\title{
In vivo experimental study on bone regeneration in critical bone defects using PIB nanogels/ boron-containing mesoporous bioactive glass composite scaffold
}

\author{
This article was published in the following Dove Press journal: \\ International Journal of Nanomedicine \\ 22 January 2015 \\ Number of times this article has been viewed
}

\author{
Xiaohui Chen ${ }^{1,2, *}$ \\ Yanbing Zhao ${ }^{3, *}$ \\ Shinan Geng ${ }^{3}$ \\ Richard J Miron' \\ Qiao Zhang' \\ Chengtie $\mathrm{Wu}^{4}$ \\ Yufeng Zhang ${ }^{1,2}$ \\ 'State Key Laboratory Breeding Base \\ of Basic Science of Stomatology \\ (Hubei-MOST) and Key Laboratory of \\ Oral Biomedicine Ministry of Education, \\ School and Hospital of Stomatology, \\ Wuhan University, People's Republic \\ of China; ${ }^{2}$ Department of Dental \\ Implantology, School and Hospital \\ of Stomatology, Wuhan University, \\ People's Republic of China; ${ }^{3} \mathrm{National}$ \\ Engineering Research Center for \\ Nanomedicine, College of Life Science \\ and Technology, Huazhong University \\ of Science and Technology, Wuhan, \\ People's Republic of China; ${ }^{4}$ State \\ Key Laboratory of High Performance \\ Ceramics and Superfine Microstructure, \\ Shanghai Institute of Ceramics, Chinese \\ Academy of Sciences, Shanghai, \\ People's Republic of China \\ *These authors contributed equally \\ to this work
}

Correspondence: Chengtie Wu State Key Laboratory of High Performance Ceramics and Superfine Microstructure, Shanghai Institute of Ceramics, Chinese Academy of Sciences, Shanghai 200050,

People's Republic of China

Email chengtiewu@mail.sic.ac.cn

Yufeng Zhang

The State Key Laboratory Breeding Base of Basic Science of Stomatology (Hubei-MOST) \& Key Laboratory of Oral Biomedicine Ministry of Education, School \& Hospital of Stomatology, Wuhan University, 237 Luoyu Road, Wuhan 430079, People's Republic of China Email zyf@whu.edu.cn
Purpose: In the present study, the fabrication of novel p(N-isopropylacrylamide-co-butyl methylacrylate) (PIB) nanogels was combined with boron-containing mesoporous bioactive glass (B-MBG) scaffolds in order to improve the mechanical properties of PIB nanogels alone. Scaffolds were tested for mechanical strength and the ability to promote new bone formation in vivo.

Patients and methods: To evaluate the potential of each scaffold in bone regeneration, ovariectomized rats were chosen as a study model to determine the ability of PIB nanogels to stimulate bone formation in a complicated anatomical bone defect. PIB nanogels and PIB nanogels/B-MBG composites were respectively implanted into ovariectomized rats with criticalsized femur defects following treatment periods of 2, 4, and 8 weeks post-implantation.

Results: Results from the present study demonstrate that PIB nanogels/B-MBG composites showed greater improvement in mechanical strength when compared to PIB nanogels alone. In vivo, hematoxylin and eosin staining revealed significantly more newly formed bone in defects containing PIB nanogels/B-MBG composite scaffolds when compared to PIB nanogels alone. Tartrate-resistant acid phosphatase-positive staining demonstrated that both scaffolds were degraded over time and bone remodeling occurred in the surrounding bone defect as early as 4 weeks post-implantation.

Conclusion: The results from the present study indicate that PIB nanogels are a potential bone tissue engineering biomaterial able to treat defects of irregular shapes and deformities as an injectable, thermoresponsive, biocompatible hydrogel which undergoes rapid thermal gelation once body temperature is reached. Furthermore, its combination with B-MBG scaffolds improves the mechanical properties and ability to promote new bone formation when compared to PIB nanogels alone.

Keywords: bone graft, boron, osteoporosis, osteoporotic defect, mesoporous bioactive graft, tissue engineering

\section{Introduction}

Osteoporosis is a worldwide disease characterized by low bone mass and poor bone strength caused by an imbalance between bone forming osteoblasts and bone resorbing osteoclasts. ${ }^{1}$ An estimated 200 million people are affected worldwide with the great majority being women. ${ }^{2}$ Reports demonstrate that within the lifetime of a patient suffering from the disease, $30 \%-50 \%$ of women and $15 \%-30 \%$ of men will suffer at minimum one osteoporotic-related fracture. ${ }^{3}$ A recent systematic review found that not only was life quality for these patients severely decreased, but the incidence of death for patients following hip fracture is at least doubled for an age-matched control without 
such fractures, and is continuously rising. ${ }^{4}$ Currently the two major pharmacological approaches are anabolic agents such as parathyroid hormone which stimulates bone formation or anti-resorptive agents including bisphosphonates, calcitonin, raloxifene, and estrogen which act by inhibiting bone resorption. ${ }^{5}$ Although much research to date focuses on the prevention of bone loss by using the abovementioned agents, less emphasis has been placed on the repair of defects following fracture while more than 1.5 million fractures occur annually in the United States alone. ${ }^{6}$

To date the replacement and repair of damaged or diseased tissues is largely driven by the field of tissue engineering which is an interdisciplinary field that draws on a multitude of disciplines including material science, cell biology, biotechnology, and biomaterials. ${ }^{7,8}$ Mesoporous bioactive glass (MBG) has been widely used as a bone replacement material due to its mesoporous structure which increases surface area and allows for preferential osteoblast adhesion, proliferation, and differentiation. ${ }^{9,10}$ Previously we have demonstrated that the incorporation of the trace element boron plays an important role in bone growth. ${ }^{9,11}$ Boron has the ability to stimulate wound healing, facilitate the release of osteo-inductive growth factors and cytokines, and increase the extracellular matrix (ECM) turnover., ${ }^{9,11,12}$ Boron-containing MBG (B-MBG) is an excellent scaffold with significantly enhanced osteogenic properties and drug delivery capability for tissue engineering compared with MBG alone. ${ }^{9}$

However, MBG as a scaffold for bone repair also has drawbacks such as inherent brittleness. ${ }^{13,14}$ In contrast, $\mathrm{p}$ (N-isopropylacrylamide-co-butyl methylacrylate) (PIB) nanogels is a temperature-sensitive cross-linking polymer and its dispersion exhibits three phase states (swollen gel, flowable sol, and shrunken gel) in accordance with temperature changes. It can be used to treat defects of irregular shapes and deformities as an injectable, thermoresponsive, biocompatible hydrogel for tissue engineering applications which undergoes rapid thermal gelation once the sol form reaches body temperature. ${ }^{15,16}$ Recently, PIB nanogels have been applied in interventional therapy for liver tumors and vascular occlusion of renal artery as a novel blood-vesselembolic material. ${ }^{15,17}$ However to date, no study has applied this new technology for the repair of bone defects. Therefore, the aim of the present study was to investigate the role of PIB nanogels as a bone substitute material for the repair of femur defects in rats. Furthermore, a new composite scaffold combining PIB nanogels with B-MBG scaffolds was fabricated and evaluated for material characterization, mechanical properties, and in vivo early bone formation in comparison to PIB nanogels alone.

\section{Material and methods Fabrication of PIB nanogels/B-MBG composite scaffold}

PIB nanogels were fabricated according to the method of precipitation polymerization, as previously described. ${ }^{17}$ B-MBG was fabricated according to the method of our previous study. ${ }^{9}$ Then, B-MBG powder was put into the PIB nanogels solution, and stirred for 48 hours at $4^{\circ} \mathrm{C}$. The mass ratios of $\mathrm{B}-\mathrm{MBG}$ powder and $\mathrm{PIB}$ nanogels in the mixture were $1: 1$ respectively.

\section{Characterization of PIB nanogels/B-MBG scaffolds}

The size of PIB nanogels were determined by dynamic light scattering (Zetasizer Nano-ZS 90; Malvern Instruments, Malvern, UK) using a helium-neon laser source $(\lambda=633 \mathrm{~nm})$ with scattering angle $90^{\circ}$. All samples were diluted with ultrapure water to $0.5 \mathrm{mg} / \mathrm{mL}$. The size was measured at the various temperatures from $25^{\circ} \mathrm{C}$ to $40^{\circ} \mathrm{C}$ with pre-equilibration for 5 minutes at each point. The dynamic viscoelastic properties of nanogel dispersions were obtained using a stresscontrolled rheometer (Kinexus; Malvern Instruments) with a parallel plate $(\Phi=40 \mathrm{~mm}$, gap set at $1.0 \mathrm{~mm})$ in the range of $20^{\circ} \mathrm{C}-50^{\circ} \mathrm{C}$ with the following parameters: shear stress $=1.0$ $\mathrm{Pa}$, heating rate $=5^{\circ} \mathrm{C} / \mathrm{min}$, and frequency $=1.0 \mathrm{~Hz}$.

\section{In vivo bone regeneration}

PIB nanogels/B-MBG and PIB nanogels were respectively implanted into the right and left critical-sized femur defect of mature osteoporotic female Wistar rats. The osteogenic properties were then evaluated by histological staining as described in the following section.

\section{Animals and surgical protocols}

Animal feeding and surgical procedures were conducted in accordance with the guidelines of Animal Care and Use Committee of Wuhan University, People's Republic of China, and authorized by the Ethics Committee at the School of Dentistry, Wuhan University. All animals were given food and water ad libitum. They were kept at room temperature under a 12 hour light/dark cycle.

\section{Preparation of ovariectoporosis model}

Ten week old mature female Wistar rats weighing between 200-300 g were used for this experiment. Ten percent 
chloralic hydras ( $4 \mathrm{~mL} / \mathrm{kg}$ body weight) was injected inside the abdomen for anesthetization. An osteoporosis model was induced by means of bilateral ovariectomy as previously described by our group..$^{10,18,19}$ Briefly, a lumbar bilateral incision was made where the ovaries were located. The ovarian artery and vein were sutured before the removal of the ovaries. The muscles and skin were respectively sutured after the ovaries had been removed.

\section{Femur defect model and scaffold implanting}

Femur defect drilling was performed under general anesthesia by intraperitoneal injection of sodium pentobarbital (40 mg/kg body weight). A linear skin incision of approximately $1 \mathrm{~cm}$ in the distal femoral epiphysis was made bilaterally and blunt dissection of the muscles was performed to expose the femoral condyle. ${ }^{20,21}$ Then, a $3 \mathrm{~mm}$ diameter anteroposterior bicortical channel was created perpendicular to the shaft axis to remove cancellous bone, by using a trephine burr at a slow speed irrigated with saline solution to avoid thermal necrosis. The drilled holes were rinsed by injection with saline solution in order to remove bone fragments from the cavity.

A total of 18 rats were used for the experiment. PIB nanogels and B-MBG/nanogels scaffolds were gently placed in the right and left defects of each femur in experimental animals. Nine animals were used to confirm the osteoporotic model as well as for the control blank defects (data not shown). Following equal implantation of each scaffold, muscles and skin were sutured respectively. Gentamycin was given by intramuscular injection to prevent postoperative infection after the incisions were closed.

At each time point of 2, 4, and 8 weeks after femur surgery, six rats were sacrificed by cervical dislocation. All femurs were then removed for histological processing.

\section{Histological preparation and staining}

The femur samples were fixed in $4 \%$ formaldehyde for 24 hours at room temperature and then flushed in water for 8 hours. Femoral condyles were decalcified in $10 \%$ ethylenediaminetetraacetic acid (EDTA) for 2 weeks, changed twice per week, and then dehydrated in a series of graded concentrations of ethanol from $70 \%$ to $100 \%$. To get a distinct view of the defect, the orientation and alignment of femurs were carefully considered during paraffin embedding. A series of slices starting at a distance of $1 \mathrm{~mm}$ proximal from the end of the growth plate with a length of $2 \mathrm{~mm}$ were chosen for evaluation. For analysis of the bone regeneration process within the defect, the central region of the $2.5 \mathrm{~mm}$ diameter defect was defined by analyzing a circular contour as area of measurement per slice, thus obtaining a consistent volume of interest and to avoid including the native bone margins.

Longitudinal serial sections, $4 \mu \mathrm{m}$ thick, were cut and mounted on polylysine-coated microscope slides. For descriptive histology, hematoxylin and eosin (H\&E) staining, Safranin O staining (Sigma S2255; Sigma-Aldrich Co., St Louis, MO, USA) and tartrate-resistant acid phosphatase staining (Sigma 387A; Sigma Aldrich Co.) were performed according to manufacturer's protocol. Specimens were examined under microscopic light by using an Olympus DP72 microscope (Olympus Corporation, Tokyo, Japan). The number of osteoclasts was counted under a light microscope. Cells containing more than three nuclei were defined as osteoclasts as previously described. ${ }^{10,22}$ To validate the results, each experiment was repeated at least three times.

\section{Evaluation of new bone formation}

Qualification of the regenerated bone was done according to H\&E staining by using Image Pro Plus 6.0 software (Media Cybernetics, Bethesda, MD, USA). Areas of newly formed bone which acquired a bluish-green stain were delineated manually and then calculated as the percentage of new bone area in total cross-sectional area ([bone area/total area] $\times 100 \%$ ) as previously described..$^{21,23-25}$ The number of osteoclasts was measured by OsteoMeasure software (OsteoMetrics, Decatur, GA, USA) following nomenclature defined by the American Society for Bone and Mineral Research. Nine randomly selected representative fields (2,048x 1,536pix) from each section were identified (original magnification $\times 10$ ) and averaged.

\section{Statistical analysis}

All statistical analysis was performed by using SPSS version 17.0 software (SPSS Inc., Chicago, IL, USA). Data (percentage of new bone formation and osteoclast number) were expressed as mean \pm standard deviation and were analyzed using one-way analysis of variance and post hoc Student's $t$-test. A 5\% $(P<0.05)$ level of significance was adopted.

\section{Results \\ Characterization of PIB nanogels/B-MBG scaffolds}

As shown in Figure 1, PIB nanogels exhibited temperaturesensitive volume phase transition. The particle size of 


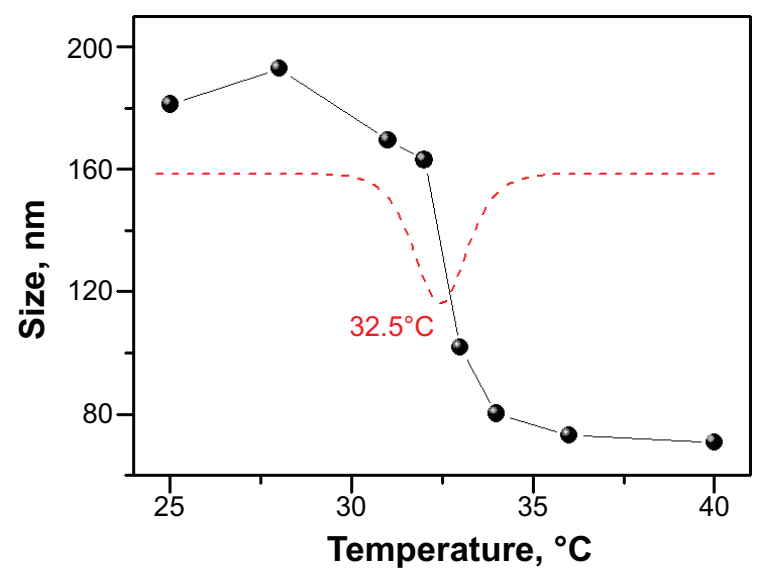

Figure I The size curve of PIB nanogels with a function of temperature, the red dash line is the differential curve of the size-temperature curve of PIB nanogels. Abbreviation: PIB, P(N-isopropylacrylamide-co-butyl methylacrylate).

nanogels decreased from about $180 \mathrm{~nm}$ at $25^{\circ} \mathrm{C}$ to $80 \mathrm{~nm}$ at $37^{\circ} \mathrm{C}$. Most notably, a sudden change of nanogels' size occurred at $32.5^{\circ} \mathrm{C}$, that is, the volume phase transition temperature. The temperature sensitive volume phase transition was attributed to the increase of the hydrophobic force between nanogels' particles in response to the increasing temperature. Therefore, concentrated PIB nanogels' dispersion exhibited different phase states depending on the temperature. At lower temperature $\left(<25^{\circ} \mathrm{C}\right)$, hydrophilic PIB nanogels swelled, and formed a swollen gel phase due to the crowded volume effect. PIB nanogels then shrank with the increasing temperature, and turned from a swollen gel phase into flowable sol phase owing to the volume reduction.

When nanogels' concentration is below $8.0 \mathrm{wt} \%$, the storage modulus (G') and the loss modulus (G') of PIB nanogels always decreased with the increasing temperature in rheological determination (Figure 2). PIB nanogels exhibited two phase states: swollen gel phase and flowable sol phase; the shrunken gel phase was not observed. The mixture of $5.0 \mathrm{wt} \%$ PIB nanogels dispersion and $6.0 \mathrm{wt} \% \mathrm{MBG}$, however, exhibited three phase states: swollen gel phase, flowable sol phase, and shrunken gel phase. Moreover, the addition of MBG improved the mechanical strength of PIB nanogels' dispersion (Figure 2).

\section{Histological observation and histological staining assessment}

Histological analysis by H\&E and Safranin O staining revealed more new bone formation in the defects filled with PIB nanogels/B-MBG scaffolds compared to PIB nanogels scaffold alone (Figures 3, 4 and 5). In both the PIB nanogels and PIB nanogels/B-MBG composite scaffolds, ECM has been formed and cells migrated inside the defects as observed by H\&E staining at 2 weeks (Figure $3 \mathrm{~A}$ and D). Defects treated with PIB nanogels were however sparsely populated with fibrous tissue along with a clear border of native bone (Figure 3A). On the other hand, signs of newly formed irregular bone trabeculae can be seen in the PIB nanogels/ B-MBG groups (Figure 3D). At 4 weeks, the density of the ECM had increased and extensive newly formed bone was observed (Figures 3B, E). Furthermore, the formation of new blood vessels randomly dispersed throughout the defect area was also observed (Figures 3B, E). The new bone of the PIB nanogels/B-MBG groups demonstrated significantly higher levels of new bone formation at 4 weeks (Figure 4). At 8 weeks, bone islands were observed in the center of defects with abundant bone formation peripherally. As compared with PIB nanogels scaffolds, significantly higher

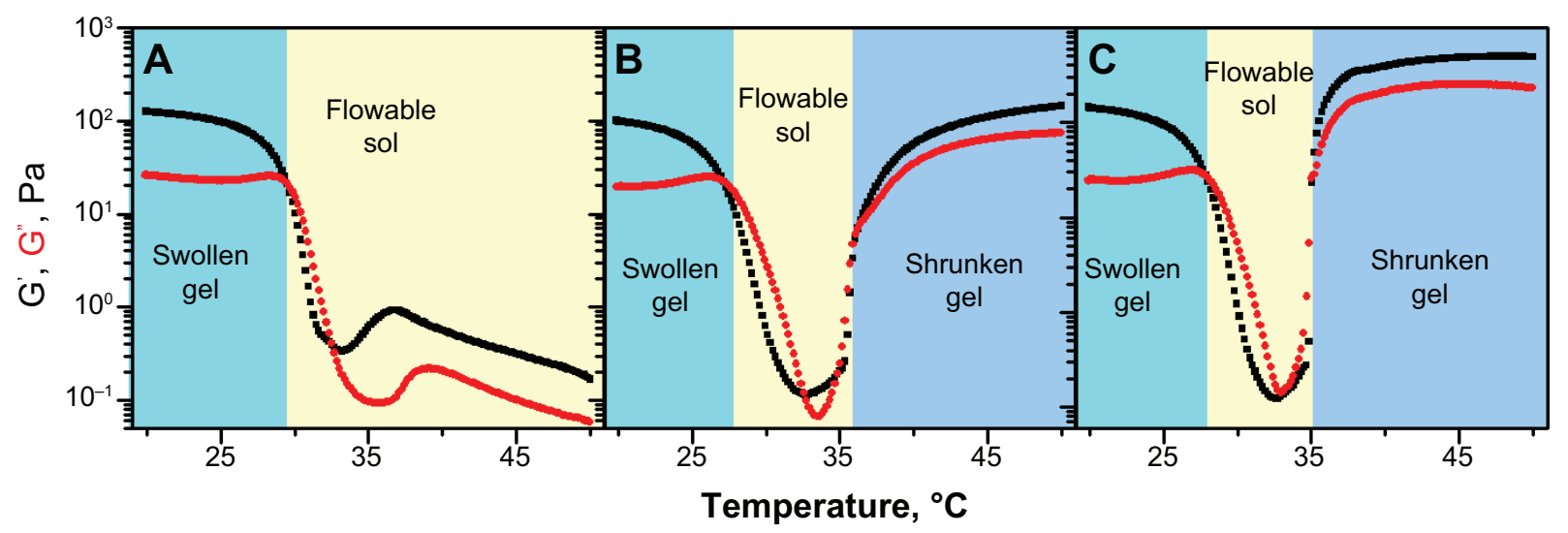

Figure 2 The dynamic viscoelastic comparison of the mixture of PIB nanogels with B-MBG.

Notes: (A) 8.0 wt\% PIB nanogels dispersion; (B) the mixture of PIB nanogels with B-MBG, the concentration of PIB nanogels and B-MBG was 5.0 wt\% and 6.0 wt\%; (C) the mixture of PIB nanogels with B-MBG, the concentration of PIB nanogels and B-MBG was 5.0 wt\% and 10.0 wt\%.

Abbreviations: PIB, $\mathrm{P}(\mathrm{N}$-isopropylacrylamide-co-butyl methylacrylate); $\mathrm{B}-\mathrm{MBG}$, boron-containing mesoporous bioactive glass; wt, weight. 

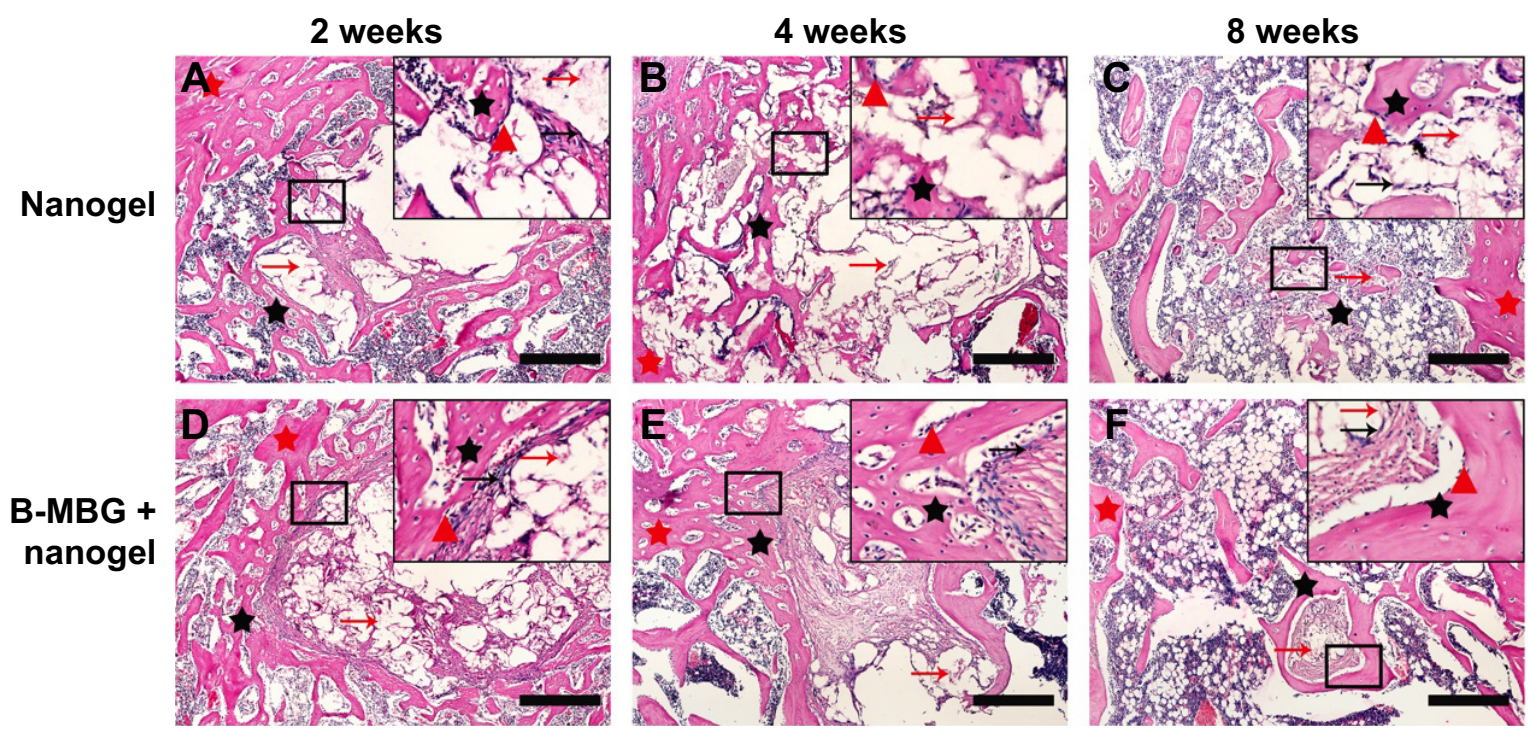

Figure 3 H\&E staining of PIB nanogels alone $(\mathbf{A}-\mathbf{C})$ and B-MBG/nanogels (D-F) at 2, 4, and 8 weeks post-implantation (bar =100 $\mu \mathrm{m})$.

Notes: Red arrow, residual material; black arrow, fibroblasts; red triangle, osteoblasts; red star, old bone; black star, new bone.

Abbreviations: PIB, P(N-isopropylacrylamide-co-butyl methylacrylate); B-MBG, boron-containing mesoporous bioactive glass; H\&E, hematoxylin and eosin.

new bone tissue was observed in the critical sized defects of PIB nanogels/B-MBG composite scaffold at 8 weeks (Figure 4).

Osteoclastic resorption was evaluated by tartrate-resistant acid phosphatase staining. At 2 weeks, shuttle shaped osteoclasts were not observed in the defects either peripherally or centrally indicating that bone remodeling was not initiated

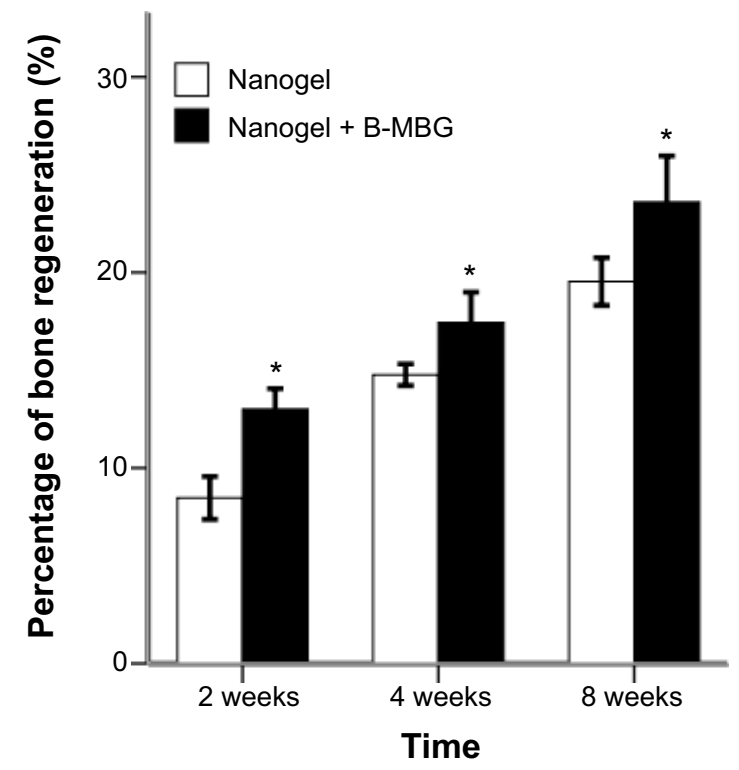

Figure 4 Early bone regeneration properties of PIB nanogels versus B-MBG/ nanogels scaffolds at 2,4 , and 8 weeks as depicted from histological analysis.

Notes: PIB nanogels containing the incorporation of B-MBG demonstrated significantly more early bone formation at all times $\left({ }^{*} P<0.05\right)$.

Abbreviations: PIB, $\mathrm{p}(\mathrm{N}$-isopropylacrylamide-co-butyl methylacrylate); B-MBG, boron-containing mesoporous bioactive glass.
(Figure 6A and D). At 4 weeks, osteoclasts were found lining the surface of the osteoporotic trabeculae. No significant difference was observed between both groups at 2 or 4 weeks (Figure 7). At 8 weeks, a gradual increase in osteoclast number was observed in both groups and a significantly lower number of osteoclasts was found on B-MBG/nanogels scaffolds when compared to control nanogels (Figure 7).

\section{Discussion}

Although a broad range of bone grafting materials has been used for tissue repair application, to date no ideal bone substitute material exists. Natural polymer-based materials are excellent scaffolds for cell attachment and growth due to their tissue-like water content and structure stability, however their weak mechanical properties make them susceptible to pressure when implanted into bone defects. ${ }^{26-28}$ Biodegradable synthetic polymer-based materials can readily be manufactured into specific shapes with relatively high mechanical strength, but they are usually hydrophobic and lack biocompatibility. ${ }^{28,29}$ Ceramic-based materials mimic the natural bone mineral, ${ }^{28,30,31}$ have revealed good bone-bonding ability $^{32}$ and osteoconductivity, ${ }^{30,31}$ however their fabrication procedures are more complex and have the inherent brittleness which limits their use in bone replacement procedures. ${ }^{28}$ In order to overcome the disadvantages of either of these materials, many composite scaffolds have been fabricated. The ideal composite should combine optimum properties such as biocompatibility, bioactivity, biodegradability, mesopore structure, and mechanical strength. 

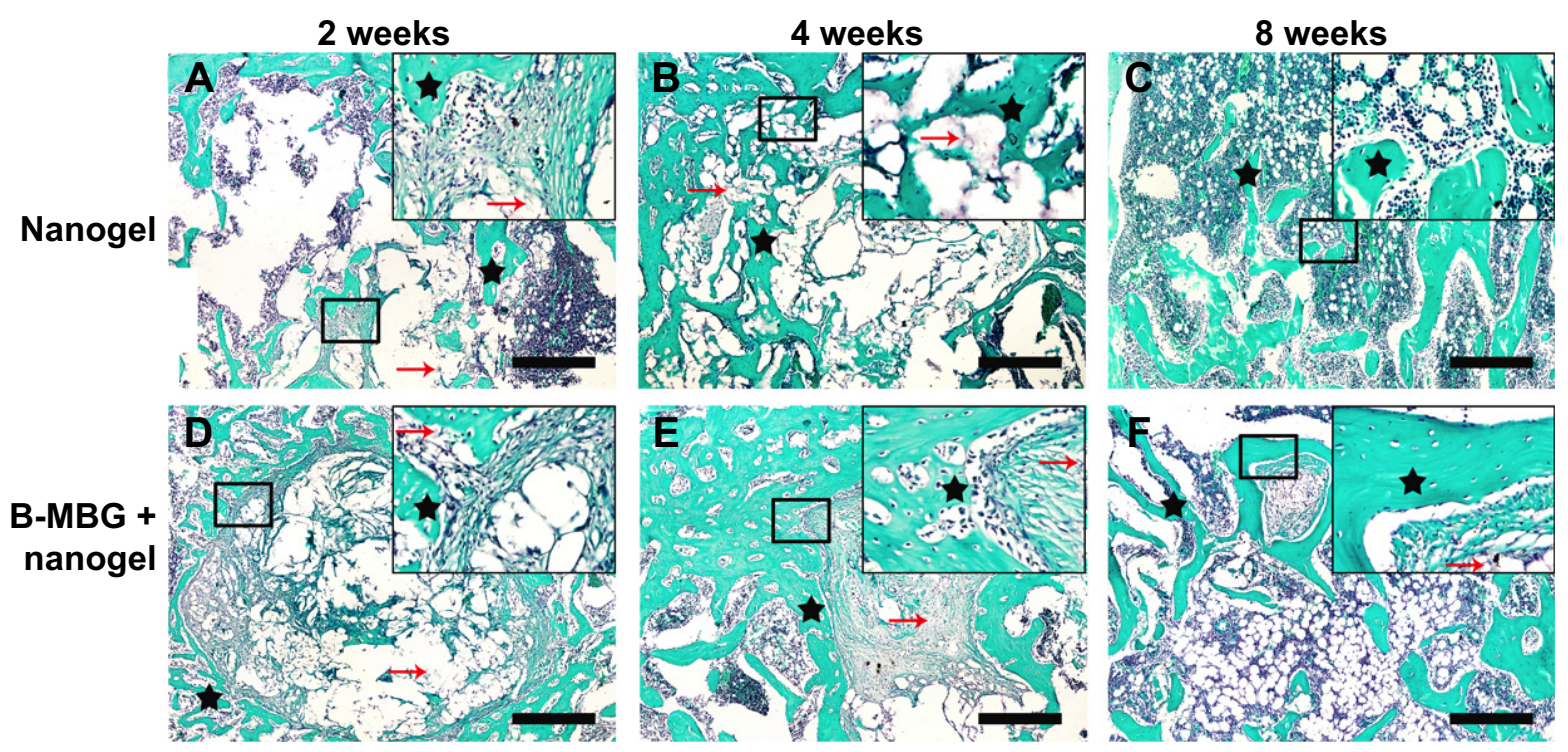

Figure 5 Safranin O of PIB nanogels alone (A-C) and B-MBG/nanogels (D-F) at 2, 4, and 8 weeks post-implantation (bar =100 $\mu \mathrm{m})$.

Notes: Black star, new bone; red arrow, residual material. (Safranin O: Sigma S2255; Sigma-Aldrich Co., St Louis, MO, USA).

Abbreviations: PIB, p(N-isopropylacrylamide-co-butyl methylacrylate); B-MBG, boron-containing mesoporous bioactive glass.

This study focuses on a novel biomaterial, PIB nanogels, and its ability to be used as a bone replacement graft. Unlike conventional biomaterials, it presents as liquid state at room temperature and solidifies at body temperature. ${ }^{15,17}$ Hydrogels are quite similar to natural living tissues, due to their ability to retain a significant amount of water or biological fluids. They are three-dimensional, hydrophilic, polymeric networks which can be used as bone substitutes and drug delivery systems. ${ }^{33}$ There are three principles for an ideal hydrogel biomaterial: 1) upon injection, it can be solidified quickly without excessive heat generation; 2) gelation temperature in vivo should be near body temperature; and 3) the gelation products should be biocompatible or bioactive to form a tight bone bonding interface. ${ }^{34} \mathrm{PIB}$ nanogels possess all three of these properties but lack some of the mechanical properties offered by ceramic scaffolds. ${ }^{15,17}$ Furthermore, the material itself undergoes material shrinkage when passing from the liquid phase at room temperature to the hardened phase at body temperature. Thus their use alone for bone tissue repair comes with certain disadvantages.
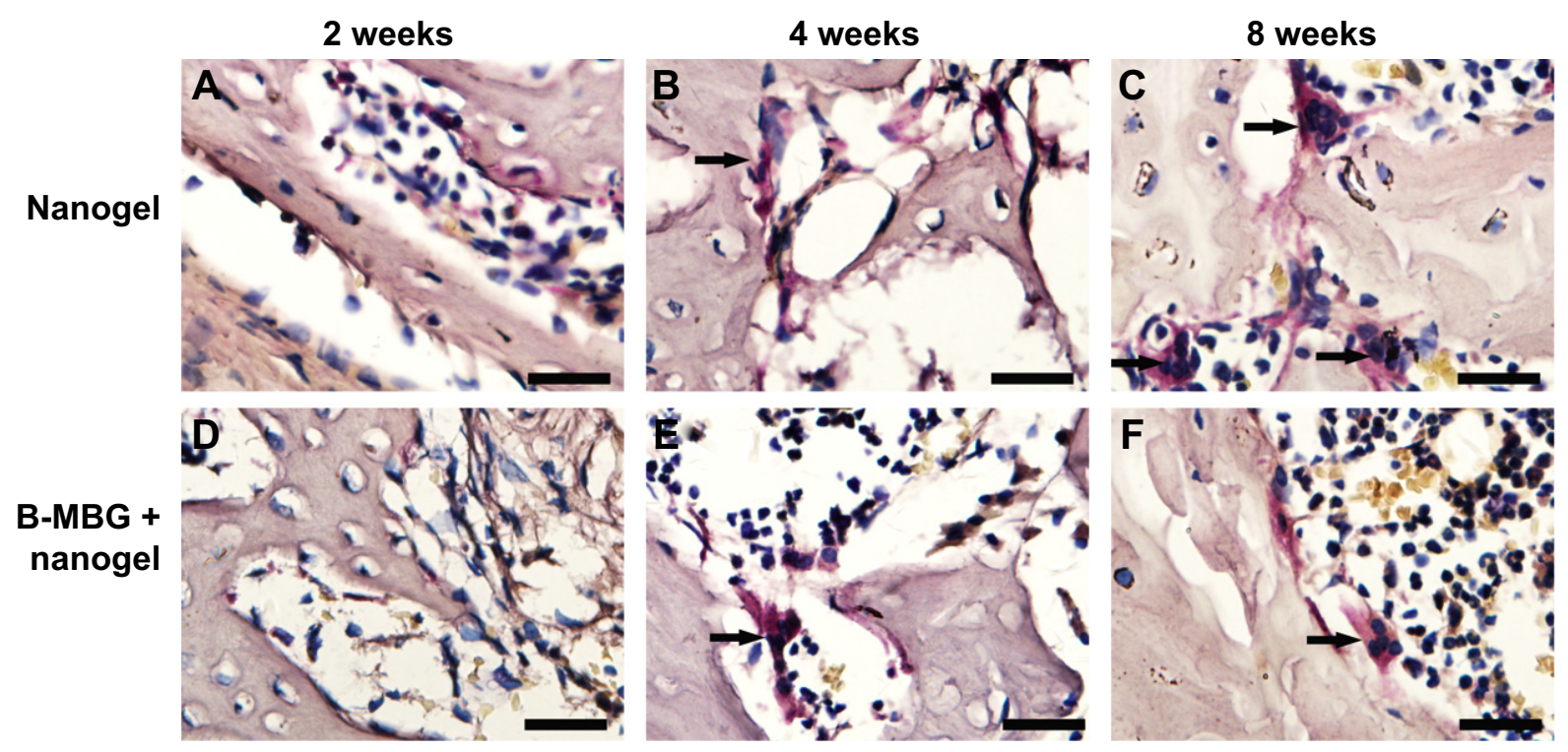

Figure 6 TRAP staining of PIB nanogels alone $(\mathbf{A}-\mathbf{C})$ and B-MBG/nanogels $(\mathbf{D}-\mathbf{F})$ at 2, 4, and 8 weeks post-implantation $($ bar $=100 \mu \mathrm{m})$.

Notes: Arrow depicts TRAP staining in multinucleated cells bordering either the scaffold surface or peripheral bone surface.

Abbreviations: TRAP, tartrate-resistant acid phosphatase; PIB, P(N-isopropylacrylamide-co-butyl methylacrylate); B-MBG, boron-containing mesoporous bioactive glass. 


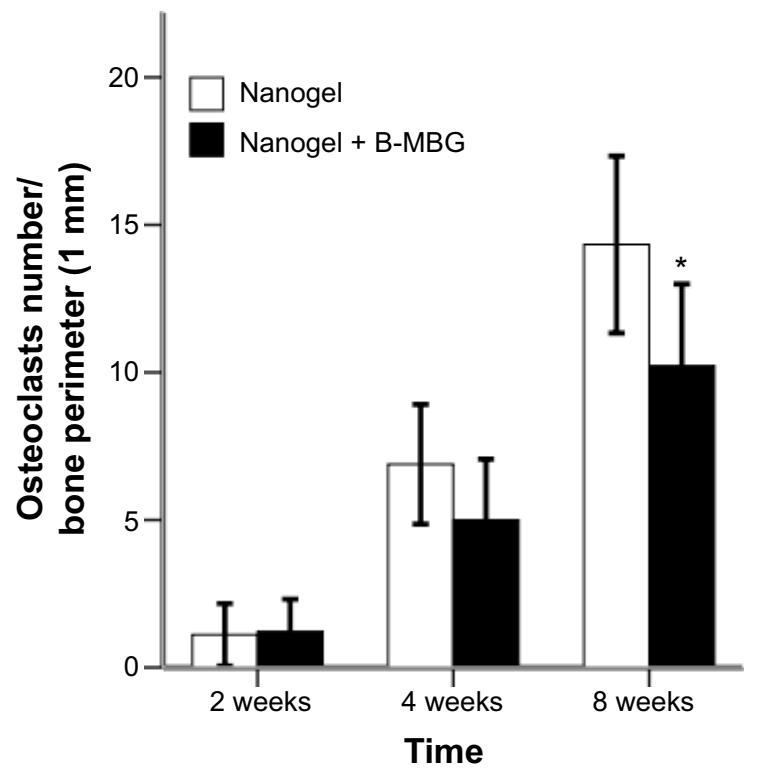

Figure 7 Number of osteoclasts from histological analysis (TRAP staining) in defects treated with either nanogels alone or B-MBG/nanogels at 2, 4, and 8 weeks postimplantation.

Note: A significantly lower number of osteoclasts was observed at 8 weeks $(* P<0.05)$. Abbreviations: TRAP, tartrate-resistant acid phosphatase; B-MBG, boron-containing mesoporous bioactive glass.

As a bioceramic material, MBG has excellent bone-like apatite-formation properties and well-ordered mesoporous channels. ${ }^{35}$ Mesoporous structures of biomaterials are of great importance for the loading and delivery of cells and drugs and can improve angiogenesis for tissue ingrowths and initiate osteoblast differentiation and bone regeneration. ${ }^{9}$ When in contact with biological fluids, bioactive glass can mechanically and chemically bond to bone by forming a carbonated hydroxyl-apatite layer on the glass surface. This bioactive bone-bonding property ensures the implant osteo-integration ${ }^{36}$ and makes nanogel one of the leading new and promising scaffold materials. ${ }^{37}$ The main disadvantage of MBG scaffolds is their brittleness and high rate of degradation. ${ }^{35}$

In the present study we explored the physical and biological properties of the PIB nanogels/B-MBG composite scaffolds. When compared to PIB nanogels alone, the incorporation of B-MBG greatly improved the mechanical strength of the PIB nanogels as well as the bone regeneration abilities of PIB nanogels. H\&E and Safranin O staining revealed significant new bone formation within the defects both peripherally and centrally in the PIB nanogels/B-MBG scaffold groups. The results demonstrate that B-MBG plays an important role in enhancing bone regeneration. Our previous study showed that B-MBG scaffolds have the ability to control the release of boron ions which promotes bone growth. ${ }^{9}$ We have previously demonstrated that osteoblasts seeded in the presence of boron demonstrate significantly higher cell proliferation and increase the bone-inducing properties by expressing higher levels of collagen 1 and Runx 2. ${ }^{9}$ Thus it is reasonable to assume that under the present study parameters, the osteoporotic healing of defects is largely driven by the release of boron which is able to stimulate osteoblast bone-forming behavior. It remains to be investigated what role boron may have on osteoclast bone-resorbing activity.

In summary, it has been demonstrated that by combining the advantages of the two biomaterials, novel PIB nanogels with B-MBG were able to efficiently generate high quantities of early bone formation in ovariectomized rats. The results from the present study indicate that PIB nanogels/B-MBG composite scaffolds have the potential to create a new class of bone repair biomaterials with consummate properties suited for bone tissue engineering.

\section{Conclusion}

The fabrication of PIB nanogels/B-MBG enhanced the mechanical properties and bone regeneration abilities compared to PIB nanogels alone. The PIB nanogels/B-MBG composite has potential future use in bone tissue engineering especially for the repair of bone defects with irregular shapes because of its ability to act as a gel at injection temperatures and solidify at body temperatures. The ordered mesoporous structure, temperature-sensitive phase transition, enhanced mechanical strength, and biocompatibility make PIB nanogels/B-MBG a desired biomaterial for bone tissue engineering.

\section{Acknowledgments}

This work was supported by Program for New Century Excellent Talents in University (NCET-11-0414), Wuhan Planning Project of Science and Technology (to XH Chen), and the funds of the National Natural Science Foundation of China (81271108 to YF Zhang).

\section{Disclosure}

The authors report no conflicts of interest in this work.

\section{References}

1. Genant HK, Cooper C, Poor G, et al. Interim report and recommendations of the World Health Organization Task-Force for Osteoporosis. Osteoporos Int. 1999;10(4):259-264.

2. Roush K. Prevention and treatment of osteoporosis in postmenopausal women: a review. Am J Nurs. 2011;111(8):26-35.

3. Seeman E, Eisman JA. 7: Treatment of osteoporosis: why, whom, when and how to treat. The single most important consideration is the individual's absolute risk of fracture. Med J Aust. 2004;180(6):298-303.

4. Abrahamsen B, van Staa T, Ariely R, Olson M, Cooper C. Excess mortality following hip fracture: a systematic epidemiological review. Osteoporos Int. 2009;20(10):1633-1650. 
5. Silva BC, Bilezikian JP. New approaches to the treatment of osteoporosis. Annu Rev Med. 2011;62:307-322.

6. Becker DJ, Kilgore ML, Morrisey MA. The societal burden of osteoporosis. Curr Rheumatol Rep. 2010;12(3):186-191.

7. Li X, Shi J, Dong X, Zhang L, Zeng H. A mesoporous bioactive glass/ polycaprolactone composite scaffold and its bioactivity behavior. J Biomed Mater Res A. 2008;84(1):84-91.

8. Mondrinos MJ, Dembzynski R, Lu L, et al. Porogen-based solid freeform fabrication of polycaprolactone-calcium phosphate scaffolds for tissue engineering. Biomaterials. 2006;27(25):4399-4408.

9. Wu C, Miron R, Sculean A, et al. Proliferation, differentiation and gene expression of osteoblasts in boron-containing associated with dexamethasone deliver from mesoporous bioactive glass scaffolds. Biomaterials. 2011;32(29):7068-7078.

10. Zhang Y, Cheng N, Miron R, Shi B, Cheng X. Delivery of PDGF-B and BMP-7 by mesoporous bioglass/silk fibrin scaffolds for the repair of osteoporotic defects. Biomaterials. 2012;33(28):6698-6708.

11. Gorustovich AA, Lopez JM, Guglielmotti MB, Cabrini RL. Biological performance of boron-modified bioactive glass particles implanted in rat tibia bone marrow. Biomed Mater. 2006;1(3):100-105.

12. Miron RJ, Zhang YF. Osteoinduction: a review of old concepts with new standards. J Dent Res. 2012;91(8):736-744.

13. Yun HS, Kim SE, Hyun YT, Heo SJ, Shin JW. Hierarchically mesoporousmacroporous bioactive glasses scaffolds for bone tissue regeneration. J Biomed Mater Res B Appl Biomater. 2008;87(2):374-380.

14. Wu C, Luo Y, Cuniberti G, Xiao Y, Gelinsky M. Three-dimensional printing of hierarchical and tough mesoporous bioactive glass scaffolds with a controllable pore architecture, excellent mechanical strength and mineralization ability. Acta Biomater. 2011;7(6):2644-2650.

15. Zhao H, Zheng C, Feng G, et al. Temperature-Sensitive poly(NIsopropylacrylamide-Co-Butyl Methylacrylate) Nanogel as an Embolic Agent: Distribution, Durability of Vascular Occlusion, and Inflammatory Reactions in the Renal Artery of Rabbits. AJNR Am J Neuroradiol. 2013;34(1):169-176.

16. Dong Y, Saeed AO, Hassan W, et al. "One-step" Preparation of Thiol-Ene Clickable PEG-Based Thermoresponsive Hyperbranched Copolymer for In Situ Crosslinking Hybrid Hydrogel. Macromol Rapid Commun. Epub 2011 Dec 5.

17. Zhao Y, Zheng C, Wang Q, et al. Permanent and Peripheral Embolization: Temperature-Sensitive $\mathrm{p}$ (N-Isopropylacrylamide-co-butyl Methylacrylate) Nanogel as a Novel Blood-Vessel-Embolic Material in the Interventional Therapy of Liver Tumors. Advanced Functional Materials. 2011;21(11):2035-2042.

18. Cheng N, Dai J, Cheng X, et al. Porous CaP/silk composite scaffolds to repair femur defects in an osteoporotic model. J Mater Sci Mater Med. 2013;24(8):1963-1975.

19. Wei L, Ke J, Prasadam I, et al. A comparative study of Sr-incorporated mesoporous bioactive glass scaffolds for regeneration of osteopenic bone defects. Osteoporosis Int. 2014;25(8):2089-2096.

20. Zhang Y, Wu C, Luo T, Li S, Cheng X, Miron RJ. Synthesis and inflammatory response of a novel silk fibroin scaffold containing BMP7 adenovirus for bone regeneration. Bone. 2012;51(4):704-713.

21. Miron RJ, Wei L, Bosshardt DD, Buser D, Sculean A, Zhang Y. Effects of enamel matrix proteins in combination with a bovine-derived natural bone mineral for the repair of bone defects. Clin Oral Investig. 2014;18(2):471-478.

International Journal of Nanomedicine

\section{Publish your work in this journal}

The International Journal of Nanomedicine is an international, peerreviewed journal focusing on the application of nanotechnology in diagnostics, therapeutics, and drug delivery systems throughout the biomedical field. This journal is indexed on PubMed Central, MedLine, CAS, SciSearch $\AA$, Current Contents $\AA /$ Clinical Medicine,
22. Fernandes JC, Wang H, Jreyssaty C, et al. Bone-protective effects of nonviral gene therapy with folate-chitosan DNA nanoparticle containing interleukin-1 receptor antagonist gene in rats with adjuvant-induced arthritis. Mol Ther. 2008;16(7):1243-1251.

23. Wang L, Fan H, Zhang ZY, et al. Osteogenesis and angiogenesis of tissue-engineered bone constructed by prevascularized beta-tricalcium phosphate scaffold and mesenchymal stem cells. Biomaterials. 2010;31(36):9452-9461.

24. Yang S, Lan L, Miron RJ, Wei L, Zhang M, Zhang Y. Variability in Particle Degradation of Four Commonly Employed Dental Bone Grafts. Clin Implant Dent Relat Res. Epub 2014 Jan 3.

25. Saulacic N, Bosshardt DD, Jensen SS, Miron RJ, Gruber R, Buser D. Impact of bone graft harvesting techniques on bone formation and graft resorption: a histomorphometric study in the mandibles of minipigs. Clin Oral Implants Res. Epub 2014 Feb 19.

26. Kim J, Kim IS, Cho TH, et al. Bone regeneration using hyaluronic acid-based hydrogel with bone morphogenic protein-2 and human mesenchymal stem cells. Biomaterials. 2007;28(10):1830-1837.

27. Park KH, Kim H, Moon S, Na K. Bone morphogenic protein-2 (BMP-2) loaded nanoparticles mixed with human mesenchymal stem cell in fibrin hydrogel for bone tissue engineering. J Biosci Bioeng. 2009; 108(6):530-537.

28. Kang SW, Kim JS, Park KS, et al. Surface modification with fibrin/ hyaluronic acid hydrogel on solid-free form-based scaffolds followed by BMP-2 loading to enhance bone regeneration. Bone. 2011;48(2): 298-306.

29. Hayashi C, Hasegawa U, Saita Y, et al. Osteoblastic bone formation is induced by using nanogel-crosslinking hydrogel as novel scaffold for bone growth factor. J Cell Physiol. 2009;220(1):1-7.

30. Li SH, De Wijn JR, Layrolle P, de Groot K. Synthesis of macroporous hydroxyapatite scaffolds for bone tissue engineering. J Biomed Mater Res. 2002;61(1):109-120.

31. Zhang R, Ma PX. Poly(a-hydroxyl acids)/hydroxyapatite porous composites for bone-tissue engineering. I. Preparation and morphology. $J$ Biomed Mater Res. 1999;44(4):446-455.

32. Tsigkou O, Hench LL, Boccaccini AR, Polak JM, Stevens MM. Enhanced differentiation and mineralization of human fetal osteoblasts on PDLLA containing Bioglass composite films in the absence of osteogenic supplements. J Biomed Mater Res A. 2007;80(4):837-851.

33. Coviello T, Matricardi P, Marianecci C, Alhaique F. Polysaccharide hydrogels for modified release formulations. J Control Release. 2007;119(1): $5-24$.

34. Zhou Z, Yang D, Nie J, Ren Y, Cui F. Injectable Poly(ethylene glycol) Dimethacrylate-based Hydrogels with Hydroxyapatite. Journal of Bioactive and Compatible Polymers. 2009;24(5):405-423.

35. Wu C, Zhang Y, Zhu Y, Friis T, Xiao Y. Structure-property relationships of silk-modified mesoporous bioglass scaffolds. Biomaterials. 2010; 31(13):3429-3438

36. Lopez-Noriega A, Arcos D, Izquierdo-Barba I, Sakamoto Y, Terasaki O, Vallet-Regi M. Ordered Mesoporous Bioactive Glasses for Bone Tissue Regeneration. Chem Mater. 2006;18(13):3137-3144.

37. Rezwan K, Chen QZ, Blaker JJ, Boccaccini AR. Biodegradable and bioactive porous polymer/inorganic composite scaffolds for bone tissue engineering. Biomaterials. 2006;27(18):3413-3431.

Journal Citation Reports/Science Edition, EMBase, Scopus and the Elsevier Bibliographic databases. The manuscript management system is completely online and includes a very quick and fair peer-review system, which is all easy to use. Visit http://www.dovepress.com/ testimonials.php to read real quotes from published authors. 